\title{
О ПРИБЛИЖЕННОЙ ОПТИМАЛЬНОСТИ СКОЛЬЗЯЩЕГО ПЛАНИРОВАНИЯ В ОДНОЙ ЗАДАЧЕ УПРАВЛЕНИЯ ПО НЕПОЛНЫМ ДАННЫМ
}

\author{
(Представил Н. Алумяэ)
}

\section{1. Введение}

Обозначив $M$ - математическое ожидание, $\lambda$ - заданная константа, ставится задача. Найти такую стратегию управления $\{u(t)\}$ с обратной связью от всех предыдущих наблюдений $u(t)=u(t, y(1), \ldots, y(t))$, которая минимизирует стоимость

$$
V(u)=M \sum_{t=1}^{N}[x(t)-\lambda]^{2},
$$

если управляемый $\{x(t)\}$ и наблюдаемый процесс $\{y(t)\}$ заданы уравнениями

$$
x(t)=u^{\mathrm{T}}(t-1) \beta_{1}(t)+\beta_{0}(t), \quad y(t)=x(t)+h(t),
$$

а вектор коэффициентов $\beta(t)=\left[\beta_{0}(t), \beta_{1}{ }^{\mathrm{T}}(t)\right]^{\mathrm{T}}$ управляемого процесса неизвестный и может изменяться согласно модели типа авторегрессии

$$
\beta(t)=\mu+\Phi[\beta(t-1)-\mu]+\alpha(t), \quad \beta(0)=\tilde{\beta} .
$$

Здесь $\mu$ - вектор среднего, Ф - матрица параметров авторегрессии. Векторы $\tilde{\boldsymbol{\beta}}, \boldsymbol{\alpha}(t), t=1, \ldots, N$ и скаляры $h(t), t=1, \ldots, N-1$ предполагаются независимыми и нормально распределенными такими, что

$$
\tilde{\boldsymbol{\beta}} \sim N(\tilde{b}, P), \quad \alpha(t) \sim I N(0, D), \quad h(t) \sim I N(0, r) .
$$

В частности, если $N=2$, то рассматриваемая задача решается [ ${ }^{1}$ ] аналитико-численным методом. В общем случае, когда $N$ - любое натуральное число, не совсем ясно как решить данную задачу ввиду сложности применения алгоритма динамического программирования. Одна возможность приближенного ее решения состоит в том, что управление строится согласно идеям скользящего планирования $\left[{ }^{2}\right]$ с одноили двухшаговым скользящим горизонтом. Понятно, что стоимость такого управления превышает стоимость оптимального управления на величину $\varepsilon \geqslant 0$. Нами в трехшаговом случае $N=3$ получена явная зависимость между величиной $\varepsilon$ и параметрами исходной задачи $\lambda, \mu, \Phi$, $\tilde{b}, P, D, r$. Построены оценки снизу и сверху стоимости оптимального управления. В многошаговом случае показано (с помощью менее точных, но простых оценок), что стратегия управления с одношаговым скользящим горизонтом является $\varepsilon$-оптимальной. 


\section{2. Оценки снизу и сверху стоимости оптимального управления}

Уточним в каком смысле понимаются используемые ниже обозначения. Запись $M^{u(t)}\{\cdot\}$, как обычно, означает. зависимость выражения под знаком математического ожидания от управления $u(t)$, а $M^{u^{*}(t)}\{\cdot\}$ означает, что в это выражение (под знаком математического ожидания) входит некоторая функция $u^{+}(t)=u(t, y(1), \ldots, y(t))$ от предыдущих наблюдений. Аналогичный смысл имеет обозначение $M^{u}{ }^{\#}{ }_{(t)}\{\cdot\}$. Введем понятия $u^{+}(t)$ - одношагового и $u^{\#}(t)$ - двухшагового управления. Назовем одношаговым управлением такое управление, которое при каждом $t=0,1, \ldots, N-1$ минимизирует функцию

$$
M^{u(t)}\left\{[x(t+1)-\lambda]^{2} / y(1), \ldots, y(t)\right\},
$$

и двухшаговым такое управление, которое при $t=0,1, \ldots, N-2$ минимизирует

$$
\begin{gathered}
M^{u(t)}\left\{[x(t+1)-\lambda]^{2}+M^{u^{*}(t+1)}\left\{[x(t+2)-\lambda]^{2} / y(1), \ldots\right.\right. \\
\ldots, y(t+1)\} / y(1), \ldots, y(t)\},
\end{gathered}
$$

а при $t=N-1-$ функцию

$$
M^{u(N-1)}\left\{[x(N)-\lambda]^{2} / y(1), \ldots, y(N)\right\} .
$$

Рассмотрим трехшаговую задачу $N=3$. С помощью элементарных преобразований из принципа оптимальности Беллмана можно вывести, что стоимость оптимального управления $V^{*}$ удовлетворяет неравенству

$$
V^{*} \leqslant M^{u^{\#}}{ }^{(0)}\left\{[x(1)-\lambda]^{2}+M^{u^{*}(1)}\left\{[x(2)-\lambda]^{2} / y(1)\right\}\right\}+\hat{R} \equiv \hat{V}
$$

где

$$
\hat{R}=\sup _{u(0)} M^{u(0)} M^{u^{+}(1)}\{L / y(1)\}
$$

$L-$ равно (2) в точке минимума, т. е.

$$
L=M^{u^{*}(2)}\left\{[x(3)-\lambda]^{2} / y(1), y(2)\right\} .
$$

Первое слагаемое правой части неравенства (3) $M^{u^{\#}}{ }_{(0)}\{\cdot\}$ измеряет стоимость оптимального управления в двухшаговой задаче. Ее можно найти как в $\left.{ }^{1}\right]$. Поэтому $\hat{V}$ может служить в качестве оценки сверху стоимости оптимального управления в трехшаговой задаче при условии, что $\hat{R}<\infty$, и удается найти явную зависимость между $\hat{R}$ и параметрами исходной задачи. В разделе 4 такая зависимость получена.

С помощью очевидных неравенств из принципа оптимальности Беллмана нетрудно вывести следующую оценку снизу

$$
V^{*} \geqslant M u^{\#}{ }^{(0)}\left\{[x(1)-\lambda]^{2}+M^{u^{*}(1)}\left\{[x(2)-\lambda]^{2} / y(1)\right\}\right\}+\check{R} \equiv \check{V}
$$

где

$$
\check{R}=\inf _{u(0)} M^{u(0)} \inf _{u(1)} M^{u(1)}\{L / y(1)\}
$$




\section{3. Качество одно- и двухшагового управления}

Пусть $V^{\#}$ - стоимость двухшагового управления. Тогда из построения оценок $\hat{V}, \check{V}$ ясно, что

$$
V^{\#} \leqslant V^{*}+\varepsilon
$$

где $\varepsilon=\hat{R}-\check{R}$

т. е. стратегия двухшагового управления является $\varepsilon$-оптимальной.

Очевидно, что

$$
\begin{gathered}
M^{u^{+}(0)}[x(1)-\lambda]^{2}+\hat{R}_{1} \leqslant M^{u^{\#}}{ }_{(0)}^{\#}\left\{[x(1)-\lambda]^{2}+M^{u^{+}(1)}\left\{[x(2)-\lambda]^{2} / y(1)\right\}\right\} \leqslant \\
\leqslant M^{u^{+}(0)}[x(1)-\lambda]^{2}+\check{R}_{1} .
\end{gathered}
$$

Здесь

$$
\hat{R}_{1}=\sup _{u(0)} M^{u(0)} M^{u^{*}(1)}\left\{[x(2)-\lambda]^{2} / y(1)\right\}
$$

а $\check{R}_{1}$ вычисляется по той же формуле, если в ней sup заменить на inf. Пусть $V^{+}-$стоимость одношагового управления. Тогда

$$
V^{+} \leqslant V^{*}+\varepsilon_{1}
$$

где $\varepsilon_{1}=\hat{R}_{1}-\check{R}_{1}+\varepsilon$.

\section{4. Явные функции для вычисления оценок}

Целью данного раздела является определение явной зависимости между величинами $\hat{R}, \stackrel{R}{R}, \hat{R}_{1}, \check{R}_{1}$ и исходными параметрами $\lambda, \mu, \Phi, \widetilde{b}$, $P, D, r$ решаемой задачи.

Введем следующие обозначения векторов средних

$$
\begin{aligned}
& a=M\{\beta(3) / y(1), y(2)\}, \quad b=M\{\beta(3) / y(1)\}, \quad c=M \beta(3), \\
& g=M\{\beta(2) / y(1)\}, \quad e=M \beta(2)
\end{aligned}
$$

и матриц ковариаций

$$
\begin{aligned}
& A=\operatorname{cov}\{\beta(3) / y(1), y(2)\}, \quad B=\operatorname{cov}\{\beta(3) / y(1)\}, \quad C=\operatorname{cov} \beta(3), \\
& G=\operatorname{cov}\{\beta(2) / y(1)\}, \quad E=\operatorname{cov} \beta(2) \text {, } \\
& W=\operatorname{cov} \beta(1) \text { : }
\end{aligned}
$$

Если $r>0$, а управления $u(0), u(1, y(1))$ ограниченные (данное условие проверяется в разделе (6)), то с помощью уравнений фильтрации и прогнозирования $\left[{ }^{3}\right]$ можно убедиться в справедливости следующих соотношений между средними

$$
\begin{aligned}
& g=e+\bar{T} w(1), \quad b=\mu+\Phi(g-\mu), \\
& a=b+T w(2)=c+\Phi \bar{T} w(1)+T w(2)
\end{aligned}
$$

и ковариациями

$$
\begin{gathered}
G=E-\bar{T} \bar{T}^{\mathrm{T}}, \quad B=D+\Phi G \Phi^{\mathrm{T}}, \\
A=B-T T^{\mathrm{T}} .
\end{gathered}
$$


Здесь $w(1), w(2)$ - независимые нормально распіределенные случайные величины такие, что $w(i) \sim N(0,1)$;

$$
\bar{T}=\Phi W z / \sqrt{r+z^{\mathrm{T}} W z} ; \quad z=\left[1, u^{\mathrm{T}}(0)\right]^{\mathrm{T}} .
$$

Функция $T$ определяется по той же формуле, что и $\bar{T}$, если в ней $W$ и $u(0)$ заменить на $G$ и $u(1)$ соответственно. Ниже индексами 0 и 1 обозначим соответственно первую и все остальные координаты рассматриваемого вектора. В случае матриц $A, B, \ldots$ используем аналогичные обозначения. Например,

$$
a=\left(\begin{array}{l}
a_{0} \\
a_{1}
\end{array}\right), \quad A=\left(\begin{array}{ll}
A_{0} & A_{10}^{\mathrm{T}} \\
A_{10} & A_{1}
\end{array}\right) .
$$

Формулы для $\hat{R}_{1}, \check{R}_{1}$. Для удобства предположим, что оценки $g_{0}$ и $g_{1}$ независимы, т. е. $\left|G_{10}\right|=0$, а матрица $G_{1}$ положительно определена. В таком случае несложно показать, что

$$
\Pi \equiv M^{u^{*}(1)}\left\{[x(2)-\lambda]^{2} / y(1)\right\}=G_{0}+\frac{\left(g_{0}-\lambda\right)^{2}}{1+g_{1}^{\mathrm{T}} G_{1}^{-1} g_{1}} .
$$

K сожалению, математическое ожидание $M^{u(0)} \Pi(w(1))$ не выражается с помощью элементарных функций - оно зависит от интеграла вероятности комплексного аргумента $\left[{ }^{1}\right]$, что не позволяет вывести явных и обозримых формул для вычисления $\hat{R}_{1}, \check{R}_{1}$. Такие формулы можно получить, если вместо функции П(w(1)) рассматривать ее линейную аппроксимацию в окрестности точки $М w(1)=0$. В таком случае

$$
M^{u(0)} \Pi \approx G_{0}+\frac{\left(e_{0}-\lambda\right)^{2}}{1+e_{1}^{\mathrm{T}} G_{1}^{-1} e_{1}} \equiv R(u(0))
$$

c погрешностью аппроксимации не превышающей величину $\varrho|\bar{T}|^{2}$. Здесь @, $\bar{T}$ - ограниченные по $u(0)$ функции. В зависимости от конкретных параметров функция $R(u(0))$ (точнее, $M^{u(0)} \Pi$, как показал численный эксперимент) либо

(Г.1) монотонно возрастает (при одновременном изменении всех координат вектор-аргумента) на интервале $(-\infty, 0]$ и убывает на интервале $[0, \infty)$, либо

(Г.2) монотонно убывает (по длине вектор-аргумента) на интервале $(-\infty, 0]$ и возрастает на интервале $[0, \infty)$.

Условия, когда имеет место (Г.1) или (Г.2), довольно громоздкие. Но в случае одномерного управления из условия выпуклости функции $R(u(0))$ вблизи точки $u(0)=0$ можно вывести, что случай (Г.1) имеет место, если

$$
\frac{\left(\Phi_{0} W_{0}\right)^{2}}{r+W_{0}}<\left(e_{0}-\lambda\right)^{2} \Phi_{1}^{2} W_{1} \frac{e_{1}^{2}}{\left(e_{1}^{2}+E_{1}\right)^{2}}
$$

например, $\Phi_{0}=0$. Если выполняется обратное к (6) неравенство, например, $\Phi_{1}=0$, то имеет место (Г.2).

Предположим (Г.1), тогда

$$
\hat{R}_{1} \approx R(0), \quad \check{R}_{1} \approx \lim _{u(0) \rightarrow \infty} R(u(0)),
$$

в противном случае (Г.2) мы должны поменять местами правые части этих уравнений. 
Формула для $\stackrel{i}{R}$. Предположим, что матрица $A_{1}$ положйтльно определена и $\left|A_{10}\right|=0$. Аппроксимируя $L=L(w(1)$, w(2)) из (4) линейной функцией в окрестности точки $M w(1)=M w(2)=0$, выражаем

$$
M^{u(0)} \inf _{u(1)} M^{u(1)}[L / y(1)] \approx \inf _{u(1)}\left\{A_{0}+\frac{\left(c_{0}-\lambda\right)^{2}}{1+c_{1}^{\mathrm{T}} A_{1}^{-1} c_{1}}\right\} .
$$

Обозначим функцию в фигурных скобках через $R(u(0), u(1))$. Предположим, что она удовлетворяет условиям (Г.1) или (Г.2) по обоим аргументам. В первом случае

$$
\check{R} \approx \lim _{u(0) \rightarrow \infty} \lim _{u(1) \rightarrow \infty} R(u(0), u(1))
$$

во втором случае

$$
\check{R} \approx R(0,0)
$$

Формула для $\hat{R}$. В данном случае найти математическое ожидание $M^{u(0)}\left\{M^{u^{+}(1)}[L / y(1)]\right\}$ от выражения в фигурных скобках особенно трудно, так как управление на втором шаге зафиксировано одношаговым. Но, используя принятую выше аппроксимацию, получим

$$
M^{u(0)} M^{u^{+}(1)}\{L / y(1)\} \approx R(u(0), \bar{u}) .
$$

Здесь $\bar{u}=M^{u(0)} u^{+}(1)$ - усредненное управление

$$
\bar{u}=-\left[e_{1} e_{1}^{\mathrm{T}}+G_{1}\right]^{-1} e_{1}\left(e_{0}-\lambda\right),
$$

которое входит в выражение для $R$ через матрицу ковариации $A=$ $=A(\bar{u})$. Ниже для сокращения записи такие матрицы ковариации обозначены через $\bar{A}$. Очевидно, что в случае (Г.1)

$$
\hat{R} \approx R(0, \bar{u})
$$

а в случае (Г.2)

$$
\hat{R} \approx \lim _{u(0) \rightarrow \infty} R(u(0) ; \bar{u})
$$

Теперь для определения функции $\hat{R}, \ldots, \check{R}_{1}$ нам осталось вычислить ковариации $G(u(0)), \bar{A}(u(0)), A(u(0), u(1))$ в интересующих нас точках управления. Из элементарных преобразований следует.

1) Если $|u(0)|=0$, то $G_{0}=\Xi, G_{1}=E_{1}$, и

$$
\begin{aligned}
& \bar{A}_{0}=D_{0}+\Phi_{0}^{2} \frac{\Xi}{1+} \frac{\Xi}{r+\check{u^{\mathrm{T}} E_{1} \check{u}} \geqslant D_{0},} \\
& \bar{A}_{1}=D_{1}+\Phi_{1}\left[E_{1}-\frac{E_{1} \check{u} \check{u} E_{1}^{\mathrm{r}} E_{1}}{r+\check{\Xi}+\check{u^{\mathrm{T}} E_{1} \check{u}}}\right] \Phi_{1} \leqslant C_{1},
\end{aligned}
$$

где

$$
\Xi=D_{0}+\Phi_{0}^{2} \frac{r}{1+} \frac{r}{W_{0}} \geqslant D_{0}, \quad \check{u}=-\left[e_{1} e_{1}^{\mathrm{T}}+E_{1}\right]^{-1} e_{1}\left(e_{0}-\lambda\right) .
$$

2) Если $|u(0)|=|u(1)|=0$, то $A_{1}=C_{1}$ и

$$
A_{0}=D_{0}+\Phi_{0}^{2} \frac{\Xi}{1+} \frac{\Xi}{r} \geqslant D_{0} .
$$


3) Если $|u(0)| \rightarrow \infty$, то $G_{0} \rightarrow E_{0}$. Если к тому $|u(1)| \rightarrow \infty$, то $A_{0} \rightarrow C_{0}$. Изменив последовательность предельных переходов, получим такой же результат.

4) Если все координаты вектора $u(0)$ стремятся к бесконечности, то $G_{1} \rightarrow D_{1}$,

$$
\begin{aligned}
& \bar{A}_{0} \rightarrow D_{0}+\Phi_{0}^{2} \frac{E_{0}}{1+} \frac{E_{0}}{r+\hat{u}^{\mathrm{T}} D_{1} \hat{u}} \leqslant C_{0}, \\
& \bar{A}_{1} \rightarrow D_{1}+\Phi_{1}\left[D_{1}-\frac{D_{1} \hat{u} \hat{u}^{\mathrm{T}} D_{1}}{r+E_{0}+\hat{u}^{\mathrm{T}} D_{1} \hat{u}} \cdot\right] \Phi_{1} \geqslant D_{1},
\end{aligned}
$$

где $\hat{u}=-\left[e_{1} e_{1}^{\mathrm{T}}+D_{1}\right]^{-1} e_{1}\left(e_{0}-\lambda\right)$.

5) Если все координаты вектора $u(1)$ стремятся к бесконечности, то $A_{1} \rightarrow D_{1}$ равномерно по всем $u(0)$.

С учетом последних утверждений легко выписать функции $\hat{R}, \stackrel{R}{R}, \hat{R}_{1}$, $\check{R}_{1}$.'Таким образом, читатель легко может построить любую из оценок $\hat{V}, \check{V}, \varepsilon, \varepsilon_{1}$ в интересующем его случае (Г.1) или (Г.2). Приведем пример для случая, когда управление одномерное, коэффициент $\beta_{1}$ не изменяется $\Phi_{1}=1, D_{1}=0$, а возмущения $\left\{\beta_{0}(t)\right\}$ центрированы $\mu_{0}=0$ и некоррелированы $\Phi_{0}=0$

$$
\varepsilon_{1} \approx \frac{\lambda^{2}}{1+} \frac{\tilde{b}_{1}^{2}}{P_{1}}+\frac{\lambda^{2}}{1+} \frac{\tilde{b}_{1}^{2}}{\frac{P_{1}}{1+} \frac{\check{P}_{1}}{r+D_{0}} \frac{\lambda^{2}}{\tilde{b}_{1}^{2}}} .
$$

В общем случае выражения для оценок сравнительно громоздкие. Но, используя неравенства для ковариации (введенные в пунктах 1), 2), 4)), можно получить менее точные, но простые оценки

$$
\varepsilon_{1} \leqslant \frac{\left(e_{0}-\lambda\right)^{2}}{1+e_{1}^{\mathrm{T}} E_{1}^{-1} e_{1}}-\frac{\left(e_{0}-\lambda\right)^{2}}{1+e_{1}^{\mathrm{T}} D_{1}^{-1} e_{1}}+\frac{\left(c_{0}-\lambda\right)^{2}}{1+c_{1}^{\mathrm{T}} C_{1}^{-1} c_{1}}-\frac{\left(c_{0}-\lambda\right)^{2}}{1+c_{1}^{\mathrm{T}} D_{1}^{-1} c_{1}}
$$

в случае (Г.1) и

$$
\varepsilon_{1} \leqslant E_{0}+C_{0}-2 D_{0}
$$

в случае (Г.2).

\section{5. Общий случай}

Рассмотрим задачу минимизации стоимости

$$
V(u)=M \lim _{N \rightarrow \infty} \frac{1}{N} \sum_{t=1}^{N}[x(t)-\lambda]^{2}
$$

относительно системы уравнений (1), если коэффициенты $\{\beta(t)\}$ управляемого процесса изменяются согласно модели типа авторегрессии - скользящего среднего $\left[{ }^{4}\right]$ с нулевыми $\alpha(s)=0, s<0$ и нормально распределенными начальными значениями $\beta(s)=\tilde{\beta}_{s}, s \leqslant 0$. Случайный процесс $\{\beta(t)\}$ предполагается стационарным таким, что $\left\{\beta_{1}(t)\right\}-$ невырожденный, т. е. матрица $D_{1}$ положительно определена. Пусть для каждого $t=1,2, \ldots$ существует ограниченное одношаговое управление $u^{+}(t-1)$ (данное условие проверяется в разделе 6 ). Тогда в силу ста- 
ционнарности прроцесса $\{\beta(t)\}$ й ограниченности одношагового упр̄авления существует предел

$$
\lim _{N \rightarrow \infty} \frac{1}{N} \sum_{t=1}^{N}[x(t)-\lambda]^{2}
$$

с конечным математическим ожиданием (по эргодической теореме), а по теореме о монотонной сходимости под знаком математического ожидания получим

$$
M^{*} \frac{1}{N} \sum_{t=1}^{N}[x(t)-\lambda]^{2} \rightarrow M^{u^{*}} \lim _{N \rightarrow \infty} \frac{1}{N} \sum_{t=1}^{N}[x(t)-\lambda]^{2} \equiv V^{+} .
$$

Построив аналогичные с (7) и (8) оценки для любого $N$

$$
\begin{gathered}
\varepsilon_{1}(N) \leqslant \frac{1}{N} \sum_{t=2}^{N}\left\{\frac{\left[b_{0}(t)-\lambda\right]^{2}}{1+b_{1}^{\mathrm{T}}(t) D_{1}^{-1}(t) b_{1}(t)}-\frac{\left[b_{0}(t)-\lambda\right]^{2}}{1+b_{1}^{\mathrm{T}}(t) D_{1}^{-1} b_{1}(t)}\right\}, \text { если } \\
\varepsilon_{1}(N) \leqslant \frac{1}{N} \sum_{t=2}^{N}\left\{D_{0}(t)-D_{0}\right\}, \text { если (Г.2), }
\end{gathered}
$$

где $b(t)=M \beta(t), D(t)=\operatorname{cov} \beta(t)$, убедимся, что существует предел

$$
\varepsilon_{1}(N) \rightarrow \bar{\varepsilon}_{1}, \quad \text { при } \quad N \rightarrow \infty
$$

в силу стационарности процесса $\{\beta(t)\}$. Таким образом, $V^{+} \leqslant V^{*}+\bar{\varepsilon}_{1}$, т. е. стратегия одношагового управления $\left\{u^{+}(0), u^{+}(1), \ldots\right\}$ является $\overline{\varepsilon_{1}}$-оптимальным.

\section{6. Фильтруемость ненаблюдаемого процесса при одно- и двухшаговом управлении}

Рассмотрим трехшаговую задачу. Пусть матрицы $W_{1}, G_{1}$ положительно определены. Тогда существует единственное одношаговое управление $u^{+}(t), t=0,1$. Выражая управление

$$
u^{+}(1)=-\left[g_{1} g_{1}^{\mathrm{T}}+G_{1}\right]^{-1}\left[g_{1}\left(g_{0}-\lambda\right)+G_{10}\right]
$$

через $w(1)$ с помощью (5) убедимся, что функция $u^{+}(1, w(1))$ ограничена в том направлении, в каком оценка $g_{1}(w(1))$ не вырождается, в остальных $i$ направлениях выполняется условие

$$
M\left[u_{i}^{+}(1, w(1))\right]^{2}<\infty .
$$

Этими условиями гарантируется фильтруемость ненаблюдаемого процесса (ср. $\left[{ }^{3}\right]$, с. 505$)$ в трехшаговой задаче и то, что при условии положительной определенности матрицы $A_{1}$ существует единственное одношаговое управление $u^{+}(2)$ на последнем шаге. Показываем, что двухшаговое управление $u^{\#}(1, w(1))$ также удовлетворяет условиям фильтруемости. Оно определяется из условий

$$
S_{u}+R_{u}=0, \quad S_{u u}+R_{u u}>0,
$$

где $S_{u}, R_{u}$ - первые, $S_{u u}, R_{u u}$ - вторые производные по $u(1)$ от функции

$$
S=M^{u(1)}\left\{[x(2)-\lambda]^{2} / y(1)\right\}, \quad R=M^{u(1)}\{L / y(1)\} .
$$


Выражая $R$ из $\left[{ }^{1}\right]$ через $w(1)$, можно убедиться, что в условии $\left[{ }^{1}\right]$ существует ограниченное двухшаговое управление при любой ограниченной $w(1)$. Если $|w(1)| \rightarrow \infty$, то одно- и двухшаговое управления стремятся к одному и тому же пределу, т. е.

$$
u^{\#}(1, w(1))-u^{+}(1, w(1)) \rightarrow 0 .
$$

Поэтому двухшаговое управление ограниченное. Действительно. Умножая правую и левую части (не-) равенства (9) на $w^{-2}(1)<\infty$, получим

$$
S_{u}+w^{-2}(1) R_{u}=0, \quad S_{u u}+w^{-2}(1) R_{u u}>0,
$$

где

$$
S_{u}(w(1))=\frac{S_{u}(w(1))}{w^{2}(1)}, \quad S_{u u}(w(1))=\frac{S_{u u}(w(1))}{w^{2}(1)}
$$

- ограниченные функции.

Одношаговое управление определяется из условий

$$
S_{u}=0, \quad S_{u u}>0 .
$$

Функции $R_{u}(w(1)), R_{u и}(w(1))$ - ограничены. Поэтому (10) следует из (11).

В общем случае (если $N>3$ ) вопрос о фильтруемости при одно- и двухшаговом управлении более сложный. Связано это с тем, что матрица ковариации $\operatorname{cov}\left\{\beta_{1}(t) / y(1), \ldots, y(t-1)\right\}$ зависит от «случая», точнее, от стандартных гауссовских величин w(1), $\ldots, w(t-2)$. Но, выражая $M\{\beta(t) / y(1), \ldots, y(t-1)\}$ через w(1), $\ldots, w(t-1)$ и аппроксимируя функцию $\operatorname{cov}\left\{\beta_{1}(t) / y(1), \ldots, y(t-1)\right\}$ по $w(1), \ldots$ $\ldots, w(t-2)$ линейной функцией в окрестности точки $M w(1)=\ldots=$ $=M w(t-2)=0$, можно показать, что одношаговое управление $u^{+}(t, w(1), \ldots, w(t-1))$ существует и имеет аналогичные с $u^{+}(1, w(1))$ свойства фильтруемости. Это при условии, что матрицы $\operatorname{cov}\left\{\beta_{1}(t) / y(1), \ldots, y(t-1)\right\}, t=1,2, \ldots$ положительно определены. Согласно условиям раздела 5 это именно так: случайный процесс $\left\{\beta_{1}(t)\right\}$ предполагается невырожденным.

\section{ЛИТЕ Р А Т У Р А}

1. Тенно Р. Изв. АН ЭССР. Физ. Матем., 32, № 1, 11-18 (1983).

2. Гайцгори В. Г., Первозванский $А$. А. Автоматика и телемеханика, № 10, 93-99 (1977).

3. Липцер Р. Ш., Ширяев А. Н. Статистика случайных процессов. М., «Наука», 1974.

4. Бокс Дж., Дженкинс $Г$. Анализ временных рядов. Прогноз и управление. Вып. 1. М., «Мир», 1974.

Ннститут кибернетики

Академии наук Эстонской ССР
Поступила в редакцию 10/XII 1984

\section{R. TENNO}

\section{LIBISEVA HORISONDIGA JUHTIMISSTRATEEGIA $\varepsilon$-OPTIMAALSUSEST}

On vaadeldud mitteteadaolevate parameetritega, lineaarse, inertsivaba, diskreetse ajaga süsteemi optimaaljuhtimise ülesannet. Erijuhul, kui juhtimise horisont on vōrdne kolmega, on leitud hinnangud alt ja ülalt optimaaljuhtimise hinna jaoks. Oldjuhul on näidatud, et üheșammulisẹ libiseva horisondiga juhțimise strateegia on $\varepsilon$-opțimaalne. 


\section{$\varepsilon$-OPTIMALITY IN SLIDING PLANNING}

Consider the following control problem of minimizing

$$
V(u)=M \sum_{t=1}^{N}(x(t)-\lambda)^{2}
$$

subject to system of equations

$$
x(t)=u^{\mathrm{T}}(t-1) \beta_{1}(t)+\beta_{0}(t), \quad y(t)=x(t)+h(t),
$$

where $\{y(t)\}$ is an observable process, $h(t)$ is an independent Gaussian variable, $\beta(t)=\left\{\beta_{0}(t), \beta_{1}(t)\right\}$ is an unknown parameters vector, $u(t)$ is a feedback control from the observations, i. e. nonanticipative function

$$
u(t)=u(t, y(1), \ldots, y(t)) .
$$

1. For the case $N=3$, when $\{\beta(t)\}$ is a first order autoregressive time series, the upper and the lower bounds for value function

$$
V^{*}=\inf _{u} V(u)
$$

have been found in explicit form. when

2. For the case,

$$
V(u)=M \lim _{N \rightarrow \infty} \frac{1}{N} \sum_{t=1}^{N}(x(t)-\lambda)^{2}
$$

and $\{\beta(t)\}$ is stationary autoregressive moving-average time series, such that $\left\{\beta_{1}(t)\right\}$ is nondegenerate, it is shown, that control strategy of one-step rolling schedules $u^{+}$is $\varepsilon$-optimal, i. e.

$$
V\left(u^{+}\right) \leqslant V^{*}+\varepsilon .
$$

